\title{
BMJ Global Health The cost and intermediary cost- effectiveness of oral HIV self-test kit distribution across 11 distribution models in South Africa
}

Katleho Matsimela, ${ }^{1}$ Linda Alinafe Sande, ${ }^{2,3}$ Cyprian Mostert, ${ }^{4}$ Mohammed Majam, ${ }^{5}$ Jane Phiri, ${ }^{5}$ Vincent Zishiri, ${ }^{5}$ Celeste Madondo, ${ }^{6}$ Stephen Khama, ${ }^{6}$ Thato Chidarikire, ${ }^{7}$ Marc d'Elbée, ${ }^{2}$ Karin Hatzold, ${ }^{8}$ Cheryl Johnson, ${ }^{9}$ Fern Terris-Prestholt, ${ }^{2,10}$ Gesine Meyer-Rath ${ }^{1,11}$

To cite: Matsimela $\mathrm{K}$, Sande LA, Mostert C, et al. The cost and intermediary cost-effectiveness of oral HIV self-test kit distribution across 11 distribution models in South Africa. BMJ Global Health 2021;6:e005019. doi:10.1136/ bmjgh-2021-005019

Handling editor Seye Abimbola

- Additional supplemental material is published online only. To view, please visit the journal online (http://dx.doi.org/10. 1136/bmjgh-2021-005019)

Received 17 January 2021 Revised 29 March 2021 Accepted 31 March 2021
Check for updates

(c) World Health Organization 2021. Licensee BMJ.

For numbered affiliations see end of article.

Correspondence to Dr Gesine Meyer-Rath; gesine@bu.edu

\section{ABSTRACT}

Background Countries around the world seek innovative ways of closing their remaining gaps towards the target of $95 \%$ of people living with HIV (PLHIV) knowing their status by 2030. Offering kits allowing HIV self-testing (HIVST) in private might help close these gaps.

Methods We analysed the cost, use and linkage to onward care of 11 HIVST kit distribution models alongside the Self-Testing AfRica Initiative's distribution of 2.2 million HIVST kits in South Africa in 2018/2019. Outcomes were based on telephonic surveys of $4 \%$ of recipients; costs on a combination of micro-costing, time-and-motion and expenditure analysis. Costs were calculated from the provider perspective in 2019 US\$, as incremental costs in integrated and full costs in standalone models.

Results HIV positivity among kit recipients was $4 \%-23 \%$, with most models achieving 5\%-6\%. Linkage to confirmatory testing and antiretroviral therapy (ART) initiation for those screening positive was $19 \%-78 \%$ and $2 \%-72 \%$ across models. Average costs per HIVST kit distributed varied between $\$ 4.87$ (sex worker model) and $\$ 18.07$ (mobile integration model), with differences largely driven by kit volumes. HIVST kit costs (at \$2.88 per kit) and personnel costs were the largest cost items throughout. Average costs per outcome increased along the care cascade, with the sex worker network model being the most cost-effective model across metrics used (cost per kit distributed/recipient screening positive/confirmed positive/ initiating ART). Cost per person confirmed positive for HIVST was higher than standard HIV testing.

Conclusion HIV self-test distribution models in South Africa varied widely along four characteristics: distribution volume, HIV positivity, linkage to care and cost. Volume was highest in models that targeted public spaces with high footfall (flexible community, fixed point and transport hub distribution), followed by workplace models. Transport hub, workplace and sex worker models distributed kits in the least costly way. Distribution via index cases at facility as well as sex worker network distribution identified the highest number of PLHIV at lowest cost.

\section{WHAT IS ALREADY KNOWN? \\ $\Rightarrow$ HIV self-testing (HIVST) is the latest addition to the growing list of HIV testing options for countries with a high HIV prevalence such as South Africa. \\ $\Rightarrow$ Recent studies have shown it as a strategy that is acceptable, feasible and effective in maximising HIV testing uptake in other sub-Saharan African coun- tries with high HIV prevalence. \\ $\Rightarrow$ We collected data on the uptake, positivity, linkage to care and cost of 11 HIVST kit distribution strat- egies alongside the Self-Testing AfRica Initiative's distribution of 2.2 million test kits in South Africa from mid-2018 to mid-2019. \\ WHAT ARE THE NEW FINDINGS? \\ $\Rightarrow$ We found that HIV self-test distribution models in South Africa varied widely along four characteristics: distribution volume, HIV positivity, linkage to care and cost. \\ $\Rightarrow$ Average costs per HIVST kit distributed varied be- tween $\$ 4.87$ (sex worker model) and \$18.07 (mobile integration model), with differences largely driven by kit volumes and level of integration into already existing services. \\ $\Rightarrow$ HIVST kit costs (at \$2.88 per kit) and personnel costs were the largest cost items throughout, but the sex worker network model was the most cost-effective model across all metrics used (cost per kit distribut- ed/recipient screening positive/confirmed positive/ initiating antiretroviral therapy).}

\section{INTRODUCTION}

South Africa is the country with the most severe burden of HIV infections worldwide, with an estimated 7.8 million people living with HIV (PLHIV) and AIDS-related deaths that account for over $23 \%$ of all reported deaths in 2019. ${ }^{1}$ Further reductions of AIDSrelated deaths and HIV transmission can be facilitated by identifying PLHIV who are 


\section{WHAT DO THE NEW FINDINGS IMPLY?}

$\Rightarrow$ Policymakers making decisions about whether to add HIVST to the HIV testing strategies can use our findings to decide between models that allow large numbers of kits to be distributed quickly (models that target public spaces with high footfall, such as community and transport hub distribution models, or workplace models), those that distribute kits in the least costly way (transport hub, workplace and sex worker models), or those that identify the highest number of people living with HIV at lowest cost (distribution via index cases at facility as well as sex worker network distribution).Across models, we however found that the cost per person confirmed positive through HIVST was higher than that of standard HIV testing.

unaware of their HIV status, early diagnosis and engagement with antiretroviral therapy (ART). ${ }^{2}$ Although the last HIV household survey, in 2017, showed that $85 \%$ of all HIV-positive individuals aged between 15 and 64 years had already been diagnosed, gaps remain, for example, in reaching men, with just below $80 \%$ being diagnosed, compared with $89 \%$ of their female counterparts. ${ }^{3}$ As such, the need to intensify HIV testing remains a critical step in achieving the Joint United Nations Programme on HIV/AIDS (UNAIDS) target to diagnose $95 \%$ of PLHIV by $2030 .{ }^{4}$

The South African National Department of Health has, in recent years, developed and implemented an array of innovative HIV testing strategies, such as communitybased approaches, to complement provider-initiated HIV testing services offered at healthcare facilities in order to expand HIV testing service (HTS). HIV self-testing (HIVST), a process in which a person collects their own specimen (blood or oral fluid), performs HIV testing using an HIV rapid diagnostic test, generally in private, and interprets the result themselves either assisted or unassisted, ${ }^{5}$ has become the latest addition to the growing list of HIV testing options, with recent studies having shown it as a strategy that is acceptable, feasible and effective in maximising HIV testing uptake. ${ }^{6-8}$ More importantly, HIVST offers a much needed alternative that allows men in particular to overcome socio-structural barriers associated with perceived stigmatisation of using a clinic, a possibly unwelcoming attitude by mostly female clinic staff and by other clients, and clinic access being restricted to normal working hours. ${ }^{9}$ A recent model analysis by our team showed that, based on preliminary cost data and effectiveness data from other settings, HIVST combined with home-based testing would have the greatest impact towards the UNAIDS 95 target of all 10 testing modalities analysed, increasing the fraction of diagnosed PLHIV to $96.5 \%$ by 2030 , and would be highly cost-effective compared with currently funded HIV interventions. ${ }^{10}$

In 2017, the second phase of the Unitaid-funded SelfTesting AfRica (STAR) Initiative began to conduct HIVST implementation research in Eswatini, Lesotho and South Africa through different models of distribution which include workplace-based distribution, facility-based distribution and community-based distribution. Over 3 million HIVST kits were distributed between 2017 and 2020 across all three countries. In South Africa, HIVST distribution was mainly targeted at men given their lower historical uptake of HIV testing services; previous analysis by our team showed that the workplace model in particular was effective in reaching men who had not been tested before, or not in the last 12 months. ${ }^{11}$ Previous work by our team evaluated the cost of communitybased HIVST distribution through STAR to be, in 2019 US $\$$, between US $\$ 8.91$ and US $\$ 9.66$ per kit distributed in Malawi, US\$17.70 in Zambia, US\$14.91 in Zimbabwe and US $\$ 14.03$ in Lesotho, comparable with the cost of standard HIV testing services in each country. These estimates however did not take onward linkage and the cost of confirmatory testing for people screening positive with HIVST into account, making direct comparisons with existing. ${ }^{12-14}$ This study adds to this by presenting the cost and intermediary cost-effectiveness of distributing oral fluid-based HIVST and onward linkage to confirmatory testing and treatment services through 11 distribution models in South Africa in an effort to provide evidence for governments making decisions regarding the further scale up of self-testing after the end of the STAR initiative.

\section{METHODS \\ Description of models}

Our economic evaluation included the three main HIVST models: facility, community and workplace. Each model comprised of discrete distribution submodels that were defined as follows:

\section{Facility distribution models}

\section{Horizontal primary healthcare (antenatal care)}

As part of post-test counselling in routine HTS, women attending their first antenatal care (ANC) visit at a primary healthcare (PHC) clinic were offered HIVST kits, to take home to their current male sexual partner(s) — defined as secondary distribution. This excluded pregnant women whose sexual partners had been diagnosed HIV positive or had tested HIV negative within the previous 3 months.

\section{Horizontal PHC (index)}

HIVST kits were offered by existing PHC staff to newly diagnosed and previously known HIV-positive clients at a PHC clinic to share with their sexual partner(s) or family members who were unaware of their HIV status.

\section{Vertical PHC}

This model involved primary distribution of HIVST for on-site screening of clients attending the clinic for different services including family planning and treatment for sexually transmitted infections. This model also used distribution agents employed by implementing partners to distribute kits in the clinics, as opposed to trained lay counsellors already employed by the clinic in the horizontal models. 
Community distribution models

Fixed point

HIVST kits were distributed at preselected locations within local communities. Distribution agents set up testing tents near areas where men tend to congregate (such as hostels, taverns and brothels), provided a demonstration of HIVST kit use to a group or individuals through either video or physical demonstration, and distributed HIVST kits to consenting clients. Clients who chose to take up HIVST were given an option of selftesting in one of the small tents on-site or taking the kit home for their private use. For clients screening positive on site, confirmatory testing conducted by a professional provider was offered on-site.

\section{Mobile integration}

This model involved integrating HIVST distribution into community-based mobile HTS in mobile testing units. In comparison with facility-based services, mobile testing units are more likely to reach men. ${ }^{15}$ Similar to fixed-point distribution, clients were given the opportunity to screen on-site, and in the case of a reactive selftest, attend confirmatory testing in the mobile testing unit.

\section{Transport hub}

This model involved the distribution of HIVST kits to commuters, taxi drivers and street vendors in densely populated taxi ranks and train stations, with high foot traffic. Distribution agents provided a demonstration of HIVST kit use and offered kits to interested clients for private use off-site.

\section{Key populations}

HIVST kits were offered to sex workers and truck drivers across four provinces of Free State, Gauteng, KwaZulu Natal and Mpumalanga. This was largely not part of an established key populations programme and involved visiting key populations' hot spots such as bars, brothels, borders and truckers' resting spots among other distribution points. Kits were for recipients' own use, either on-site or off-site.

\section{Sex workers}

As part of an established sex worker health programme, HIVST kits were offered to consenting sex workers to distribute to peers who they thought might be at risk of HIV and not yet tested and who might or not be engaged in sex work themselves. Kits were not distributed to clients or for use by the sex workers themselves, and were only given to sex workers who were aware of their own status.

\section{Flexible community model}

The flexible community distribution model involved door-to-door kit distribution including distribution in marketplaces, hot spots, malls, community events and informal workplaces in communities as well as distribution as part of promoting voluntary medical male circumcision uptake. The targeted population were men and young people; kits were for their own use or to distribute to a sexual partner or partners.

\section{Workplace distribution models}

Workplace distribution was predominantly conducted in a number of male-dominated sectors such as manufacturing, mining, construction, security, petroleum and agriculture. There were two types of workplaces included: (a) larger companies that did not have formalised HIV testing programmes or had a significantly low HIV testing uptake were contacted before the distribution event for sensitisation; (b) distribution also took place more ad hoc and without prior arrangement with management to employees of smaller workplaces such as petrol stations or construction sites. We distinguished between a workplace (third-party) and a workplace (direct) distribution model, based on whether a third-party implementing organisation or a direct STAR partner was used to conduct the distribution.

\section{Survey outcomes and data analysis}

Distribution agents collected demographic information (year of birth, sex) of recipients of primary and, where relevant, secondary distribution using paper-based data collection forms or electronic tablets. Primary recipients consenting to telephonic follow-up calls provided their cellular phone number. A subset of consenting recipients was followed up telephonically with up to three calls at approximately 2-week, 4-week and 6-week time intervals post-distribution, following convenience sampling. During the call, a trained linkage officer administered a standardised questionnaire to record kit usage by the primary recipient and/or their partners as well as the HIVST result and, in the case of a positive result, whether recipients had attended confirmatory testing and initiated ART. For the mobile integration model only, outcomes data included recipients screening on-site only; linkage to care was established by record review in surrounding clinics through local HIV care organisations rather than telephonic interview. We calculated the percentages of kits whose recipients (a) were successfully called and stated that they or their partner had (b) used the kit, and for those screening positive for HIV, stated that they or their partner had linked to care for (c) confirmatory testing and (d) ART initiation. We then applied these percentages to the total number of kits distributed to estimate uptake, HIV screening positivity and linkage rates for the entire population of self-test kit recipients, allowing us to produce model-specific care cascades. Survey data were entered into Excel databases summarised by model. Statistical analysis was performed using SAS Enterprise Guide V.5.1 (Statistical Analysis Software Institute).

\section{Cost analysis}

In accordance to international costing guidelines adopted from the Global Health Cost Consortium reference $\operatorname{case}^{16}$ and using the same methods used in phase 
1 of the STAR initiative, the cost analysis was conducted in order to provide comparable results across all countries covered by the STAR initiative. ${ }^{13}$ In short, economic costs were estimated from the provider perspective and included capital cost items such as start-up training, sensitisation and equipment, and recurrent cost items such as personnel, test kits, other supplies, transportation, and building operation and maintenance. Capital costs were annualised over the 2 years' duration of the initiative's work in South Africa, using a 3\% discount rate. Since most costs were recorded in expenditure logs maintained by the implementing partners, we used a detailed expenditure analysis complemented by activitybased observations (time-and-motion analysis) and micro-costing, in particular of those models that shared resources with existing activities - the facility and mobile integration models. For these two models, incremental costs were estimated, while costs for the other models represented the full costs of implementation. Costs were estimated in 2018/2019 South African rand (ZAR) and converted to US dollars (US\$) using the period average of $14 \mathrm{ZAR}=\mathrm{US} \$ 1{ }^{17}$

We allocated resources shared across a number of models, such as the time of management and supervision staff as well as vehicles and distribution equipment based on expenditure codes that implementing staff had been trained to add to each payment requisition and invoice over the course of the project. In addition, detailed, repeated interviews with team managers over the duration of the project were conducted to fill in gaps left by the expenditure coding and add clarification.

We combined these data with micro-costing data to estimate the total cost of each model over 12 months of implementation (including capital costs that had been incurred before this 12-month period), and divided total costs by the number of HIVST kits distributed in each model during the same time period to arrive at average costs per kit distributed. Data collection time periods varied slightly between models: the workplace and transport hub models were implemented in two shorter time periods in March 2018September 2018 and September 2019, and in July 2018March 2019 and August-September 2019, respectively, which were the time periods included in the analysis. For all other models, we used resource use and outcomes 12 consecutive months: July 2018-June 2019 for the flexible community, key populations, workplace and vertical PHC models, and April 2018-March 2019 for all remaining models. While we included all start-up and preparation costs regardless of when they were accrued, our analysis of recurrent costs was restricted to time periods of full implementation, in order to include a more representative set of operations at full efficiency.

Our analysis assumed a unit price of $\$ 2$ per kit following a charitable support agreement between the Bill and Melinda Gates Foundation and the OraSure Technologies Incorporated (OraQuick HIV Self-test manufacturer). ${ }^{18}$ A marginal freight and shipping cost from the manufacturer to central warehouses in South Africa was estimated to be $\$ 0.88$ based on central expenditure records from Population Sciences International, a non-profit organisation that was mandated to distribute HIVST kits under STAR. Total costs, average cost per test kit distributed and average cost per client initiated on ART were estimated and summarised by model.

\section{Intermediary cost-effectiveness analysis}

We combined the cost and outcomes data to estimate the cost of HIVST distribution per person screening positive with a kit, per person found HIV positive in confirmatory testing and per person initiating ART. We added the cost of facility-based confirmatory testing with rapid tests based on an update to a previous analysis ${ }^{10}$ to the latter two cost estimates, and the cost of an ART initiation visit without additional testing the cost estimate per person initiating ART. ${ }^{19}$

\section{Patient and public involvement}

Patients or the public were not involved in the design, recruitment, conduct or dissemination of this study.

\section{RESULTS}

\section{Outcomes}

During the 12-month costing period (mid-2018 to mid2019), a total of 1111726 HIVST kits were distributed across the models, with $49 \%$ distributed through the flexible community model, and another $19 \%$ distributed through the workplace (third-party) model. The least number of HIVST kits, less than $1 \%$ each, were distributed through the mobile integration, horizontal PHC and vertical PHC models (online supplemental table S1).

Telephonic follow-up aimed at a response rate of at least $10 \%$ of all recipients but varied significantly across models, with a total of 52195 clients called, and 40834 clients reached (5\% and $4 \%$ of recipients, respectively). Models with the highest percentage of recipients reached were the horizontal PHC models (ANC, 64\% and index, $77 \%$ ) (online supplemental table S1). The high follow-up in these facility models was intended and was facilitated by low HIVST kit distribution volumes in these models. Conversely, follow-up for higher volume models such as the transport hubs, sex worker network and workplace (third-party) models ranged from 3\% to $30 \%$.

HIV screening positivity ranged between $4 \%$ in the third-party workplace model and $23 \%$ in the horizontal PHC (index) model (figure 1 and online supplemental table S1). The majority of models had around 5\% positivity. The proportion of recipients screening positive with an HIVST kit who reported to have obtained confirmatory testing ranged from $19 \%$ in the sex worker network model to $78 \%$ in the horizontal PHC (ANC) model (figure 1). Out of those clients with reactive HIVST who obtained confirmatory testing, between $4 \%$ (fixed point model) and $100 \%$ (vertical PHC model) reported to have initiated ART. This means that out of everyone screening positive, between $2 \%$ (fixed-point model) and $72 \%$ (horizontal PHV model, ANC) initiated ART. 


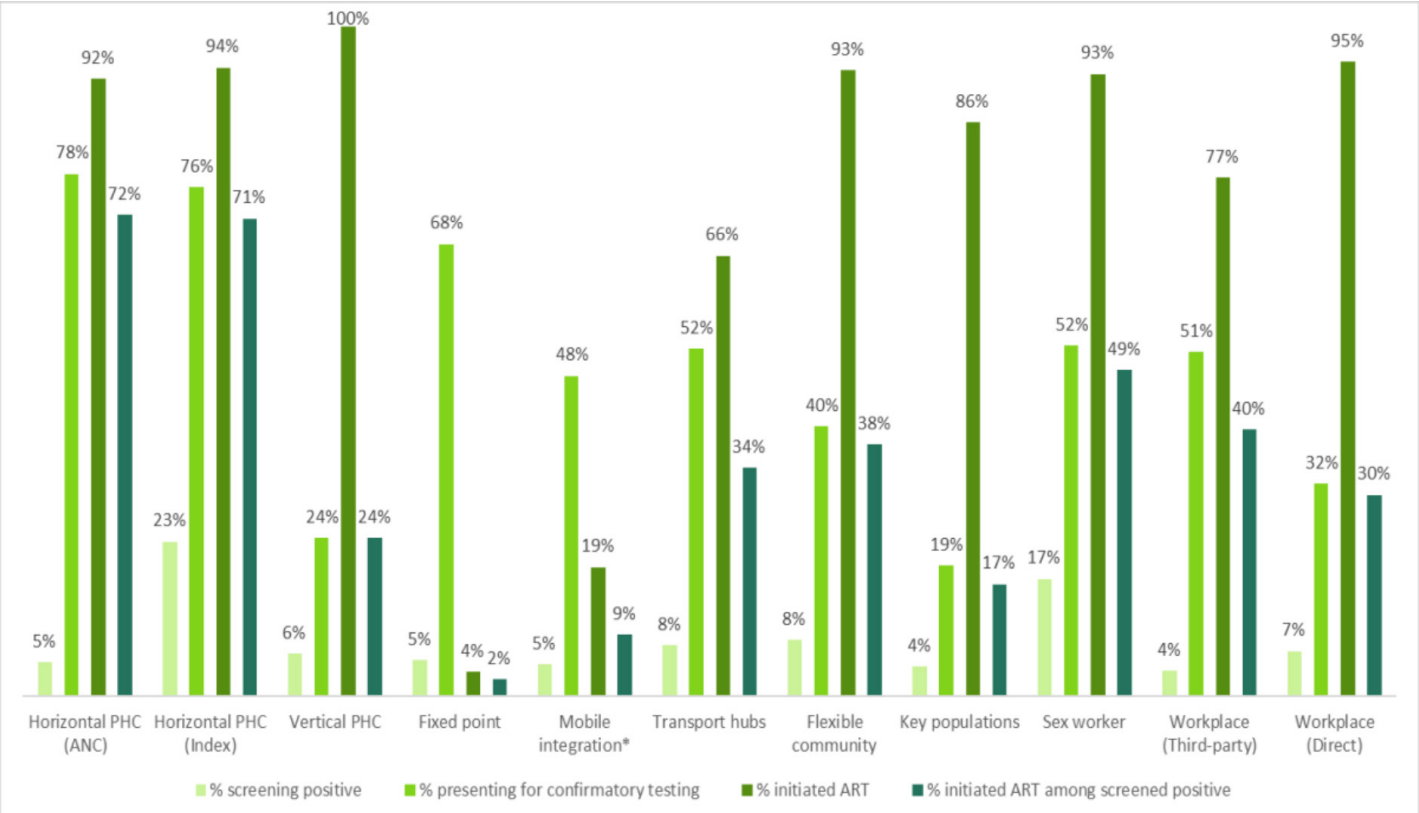

Figure 1 Care cascades by model. *Outcomes data on the mobile integration model included recipients screening on-site only; linkage to care was established by record review rather than telephonic interview, possibly biasing results downwards. ANC, antenatal care; ART, antiretroviral therapy; PHC, primary healthcare.

\section{Distribution costs}

The total cost of HIVST kit distribution varied significantly across models, ranging from $\$ 39276$ in the mobile integration model to $\$ 4003412$ in the flexible community model (table 1 ). The average cost per HIVST kit distributed varied between $\$ 4.87$ in the sex worker model and $\$ 18.07$ in the mobile integration model, with distributed kit volumes driving most of the difference in average costs, suggesting strong economies of scale (figure 2). Facility-based models exhibited higher average cost per HIVST kit distributed compared with almost all community-based and workplace-based models, with the exception of the mobile integration model (figure 2).

Recurrent costs accounted for $75 \%$ or more of total costs in all models except the key populations model (62\%) (online supplemental figure S1). Personnel and HIVST kit costs were key cost drivers across all models (online supplemental figure S1). Personnel costs accounted for more than $50 \%$ of the total cost of distribution across all PHC models, and to less than $10 \%$ in the key populations and direct workplace model. Although the unit price of an HIVST test kit before shipping remained constant at $\$ 2.00$ throughout the costing period, the total cost contribution of HIVST kits varied significantly across all models, accounting for a range between $13 \%$ in the vertical PHC model and 67\% in the workplace (direct) model (table 1).

\section{Intermediary cost-effectiveness}

When taking into account cost per intermediary outcome such as the number of recipients screening positive, confirmed positive and initiating ART, two trends were apparent.
First, cost per outcome increased by one to three orders of magnitude as a result of the inclusion of additional items such as confirmatory testing and ART initiation at a heath facility (figure 3 ). This increment in magnitude was especially influenced by the much smaller denominator defined by the HIV screening positivity, particularly in the vertical PHC, mobile integration, key populations and workplace (direct) models (online supplemental table S2). The cost per recipient screening positive ranged from $\$ 28$ in the sex worker model to $\$ 382$ in the mobile integration model; the cost per recipient confirmed positive ranged from $\$ 59$ in the sex worker model to $\$ 1229$ in the vertical PHC model; and the cost per recipient initiating on ART ranged from $\$ 112$ in the sex worker model to $\$ 5164$ in the fixed point model (online supplemental table S2). Two community models, the fixed point and the mobile integration models, had particularly steep increases between the cost per recipient confirmed positive and initiating on ART due to the large drop-offs between these two levels of care.

Second, the ranking of models from least to most costly per outcome was steered by the differences between models in both HIV positivity and onward linkage to care (table 2). Among the facility models (blue in table 2), the horizontal models were both cheaper and more cost-effective than the vertical model which employed stand-alone distribution staff, with the horizontal index testing model being the cheapest and most cost-effective facility option-in fact, the second most effective option of all 11 models, after the sex worker model, and across all outcome metrics. Among the workplace models, the 


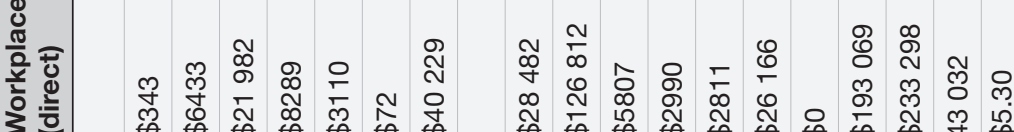

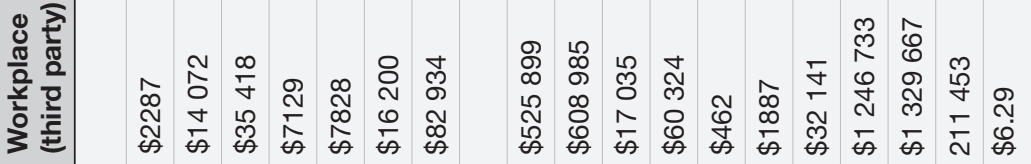

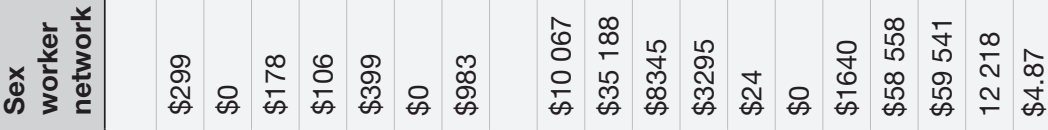

$\frac{\substack{0 \\ \frac{0}{6}}}{\frac{0}{2}}$

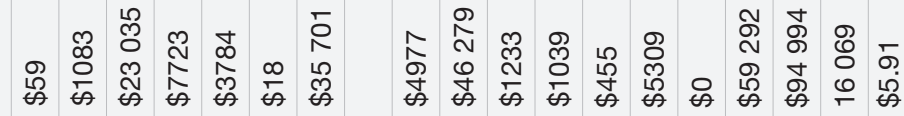

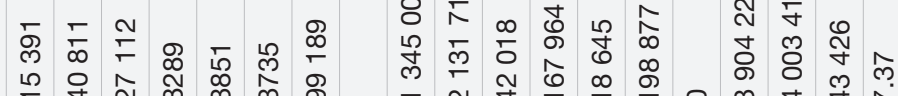

0

t⿱宀

N $\quad$ N

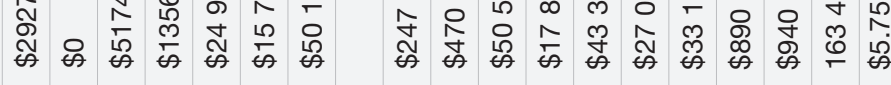

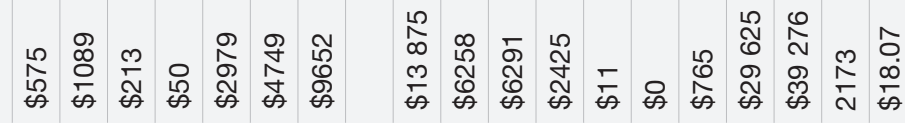

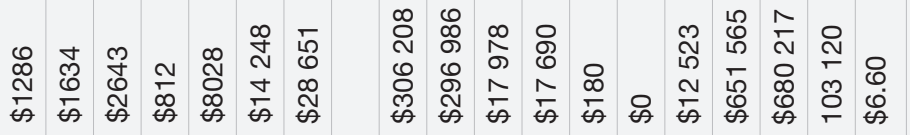

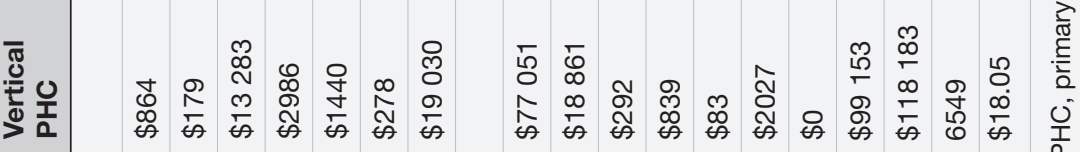

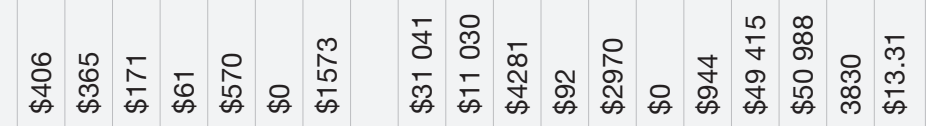

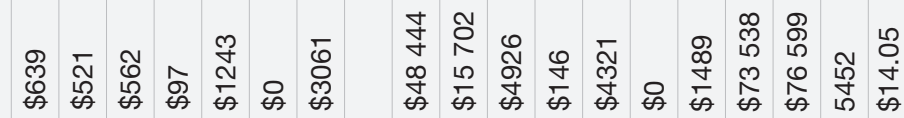




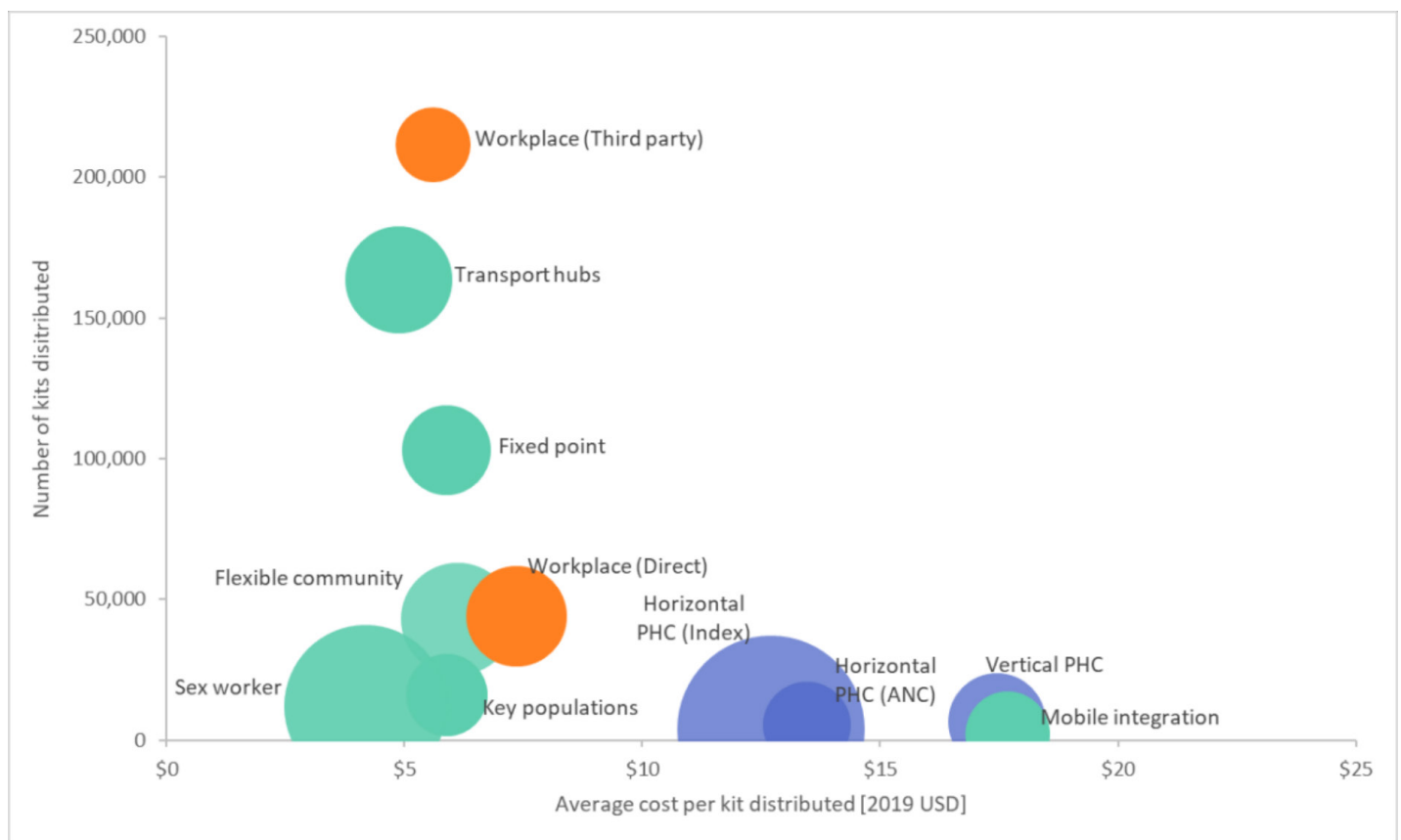

Figure 2 Relationship between distributed kit volumes by model, average cost per distributed kit and positivity (bubble size). Facility models are marked blue, community models, teal, and workplace models, orange. ANC, antenatal care; PHC, primary healthcare.

direct model was more cost-effective throughout than the model implemented by a third party, even though the former distributed more test kits.

\section{DISCUSSION}

Our analysis summarises the cost, HIV screening positivity and linkage outcomes of a wide range of HIVST distribution mechanisms in South Africa, representing almost all models currently in use in the country. These include facility-based and community-based models as well as workplace models, primary and secondary distribution, and integrated as well as standalone models. The analysis thus serves as a good evidence base on which to base decisions regarding which models should be scaled up further, and how to improve the cost-effectiveness of existing models.

The way that models rank based on different outcome metrics has implications for decision-making. If the aim is to identify the model to distribute the largest number of kits through, the choice would be the workplace and community models aimed at the general population, such as the flexible community, transport hub or fixedpoint models, while the facility and sex worker/key population models would be less attractive. (Here it is important to bear in mind that kit volumes distributed in this study were subject not only to demand but also to a number of supply side factors, including capacity and convenience, which might play out differently in

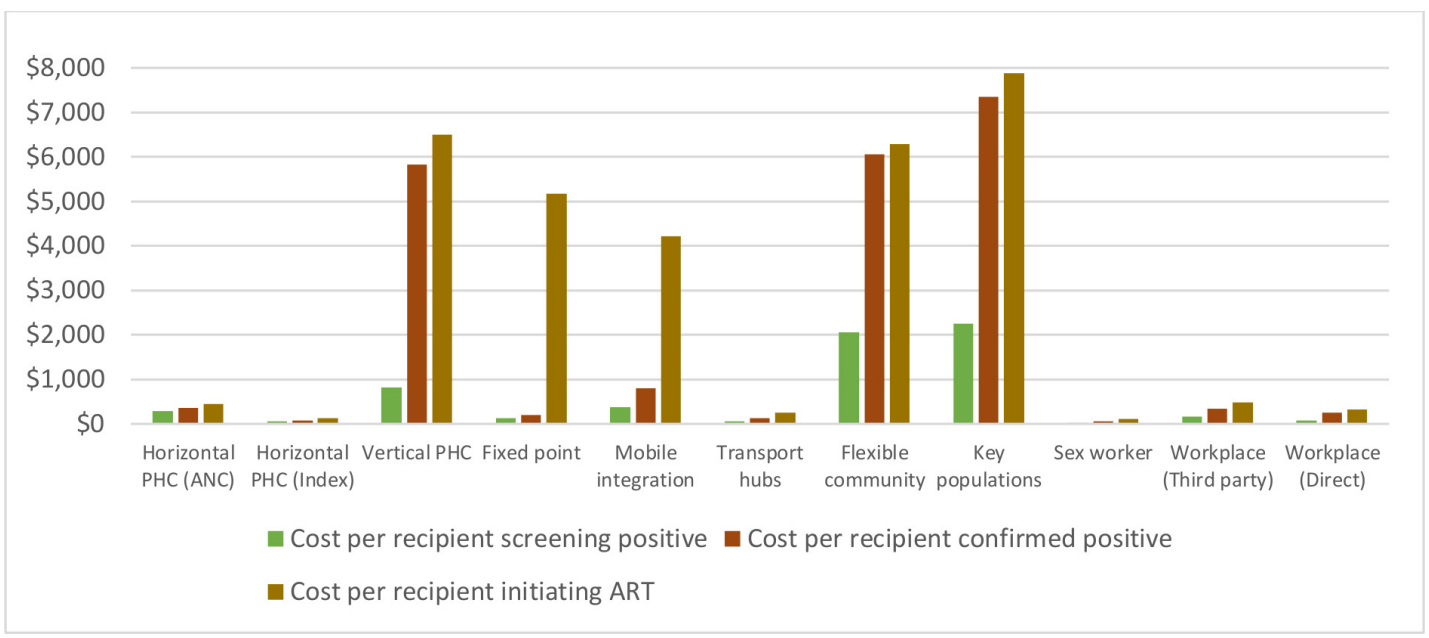

Figure 3 Cost per outcome by model. ANC, antenatal care; ART, antiretroviral therapy; PHC, primary healthcare. 
Table 2 Ranking of models by outcome

\begin{tabular}{|c|c|c|c|c|}
\hline $\begin{array}{l}\text { Number of kits } \\
\text { distributed } \\
\text { (decreasing) }\end{array}$ & $\begin{array}{l}\text { Cost per test kit } \\
\text { distributed (increasing) }\end{array}$ & $\begin{array}{l}\text { Cost per recipient } \\
\text { screening positive } \\
\text { (increasing) }\end{array}$ & $\begin{array}{l}\text { Cost per recipient } \\
\text { confirmed positive } \\
\text { (increasing) }\end{array}$ & $\begin{array}{l}\text { Cost per recipient } \\
\text { initiating ART } \\
\text { (increasing) }\end{array}$ \\
\hline Flexible community & Sex worker & Sex worker & Sex worker & Sex worker \\
\hline $\begin{array}{l}\text { Workplace (Third } \\
\text { party) }\end{array}$ & Transport hubs & Horizontal PHC (Index) & Horizontal PHC (Index) & Horizontal PHC (Index) \\
\hline Transport hubs & Workplace (Direct) & Transport hubs & Transport hubs & Transport hubs \\
\hline Fixed point & Key populations & Workplace (Direct) & Fixed point & Workplace (Direct) \\
\hline Workplace (Direct) & Flexible community & Fixed point & Workplace (Direct) & Horizontal PHC (ANC) \\
\hline Key populations & Workplace (Third party) & Workplace (Third party) & Workplace (Third party) & Workplace (Third party) \\
\hline Sex worker & Fixed point & Horizontal PHC (ANC) & Horizontal PHC (ANC) & Mobile integration \\
\hline Vertical PHC & Horizontal PHC (Index) & Mobile integration & Mobile integration & Fixed point \\
\hline $\begin{array}{l}\text { Horizontal PHC } \\
\text { (ANC) }\end{array}$ & Horizontal PHC (ANC) & Vertical PHC & Vertical PHC & Flexible community \\
\hline $\begin{array}{l}\text { Horizontal PHC } \\
\text { (Index) }\end{array}$ & Mobile integration & Flexible community & Flexible community & Vertical PHC \\
\hline Mobile integration & Vertical PHC & Key populations & Key populations & Key populations \\
\hline
\end{tabular}

Facility models are marked in different shades of blue, community models, in teal, and workplace models, in orange. (Note that the depth of shade has no significance other than to make it easier to identify and compare the position of each individual model across the rubrics).

routine implementation; additionally, there might be a demand saturation effect if the same venues were to be used regularly. ${ }^{20}$ ) If the aim is to distribute kits most efficiently and least costly, the transport hubs and workplace models would still be attractive, but so would be the sex worker model. If however the aim is to distribute kits in a way that finds the most HIV-positive people most cheaply, then secondary distribution via index cases at facility as well as integration into existing sex worker programmes and distribution to their networks is the most efficient choice. The large-volume models (workplace, transport hub or fixed-point models) achieve greater demand, largely due to the bigger footfall in busy urban thoroughfares and distribution to 'captive audiences' at urban and rural workplaces, but do so at the cost of smaller HIV positivity-in some cases augmented by lower linkage to onward care-and might be the first to saturate. The cost-effectiveness of these models might be augmented by improved targeting of population groups at higher risk of HIV acquisition.

Overall, the costs of HIVST distribution that we found across models in this study are comparable with those of community-based HIVST distribution estimated by our team, between $\$ 8.91$ and $\$ 17.70$ in Malawi, Zambia, Zimbabwe and Lesotho. ${ }^{12-14}$ We found that the costs are lower the more flexible and nimble the model is, such as all community models, and lower in models that integrate into existing services, such as in the sex worker model or the horizontal PHC models. Models that had milestone-based contracts, such as the third-party workplace and transport hub models, also achieved higher volumes more cheaply than those where this was not a possibility. Compared with the cost per positive HIV test from our previous analysis, which ranged from $\$ 4.74$ (home-based testing in an urban setting) to $\$ 17.89$ (mobile testing with mobilisation event) in $2016 \mathrm{US} \$,^{10}$ our cost per person confirmed positive $(\$ 59-\$ 1229)$ is much more expensive-pointing to the fact that identifying PLHIV will only ever get more expensive, given dwindling yield and the need for innovative methods to reach those reluctant to test.

Our study's findings stand next to a number of limitations. First, implementation was managed by a nongovernmental initiative, and both costs and outcomes might change once HIVST becomes part of routine care. Outcomes depending on HIV positivity, in particular, which, if HIV testing coverage keep up with the epidemic, will decrease over time as more PLHIV know their status, and the identification of new PLHIV will mostly be driven by HIV incidence. Second, our outcomes were based on telephonic surveys of recipients and are thus subject to a number of biases, including differential consent to being surveyed and response rates between models. Another aspect is the potential for social desirability bias in responding to survey questions which might have led people to respond positively to the question whether they had used the HIVST kit or linked to onward care, and possibly in the negative to the question what the test result was if they had not in fact used the kit. While this would have led to an overestimation of uptake and an underestimation of true screening positivity, these biases are less likely to differ by model in such a way and magnitude that our recommendations no longer hold. Third, we did not differentiate between primary and secondary distribution, given that all models with the exception of the sex worker and the horizontal PHC models used both 
methods, and recommendations for one over the other would ignore that there is often demand for both and the most efficient models seem to be those that fulfil this demand flexibly, adding kits to take to partners to every distribution of kits for the recipient's own use. Further details of these differences can be found in Sande $e t$ al (submitted to the same issue). Lastly, our analysis stopped at intermediary outcomes particular to HIVST; a full comparison with other HIV testing modalities on the one hand and other HIV interventions on the other would require the inclusion of modelled outcomes such as life-years saved and HIV infections averted as well as the consideration of the full cost of the national HIV programme, including antiretroviral treatment, which was not part of this analysis. Our recommendations, while useful for choosing between channels for HIVST distribution once a decision for HIVST scale-up has been made, do hence not help with the decision of whether or not HIVST should in fact be funded and further scaled up from a limited public HIV budget.

\section{CONCLUSION}

HIV self-test distribution models varied widely along four characteristics: distribution volume, screening positivity, linkage to onward HIV care and cost. Distribution volume was highest in models that flexibly distributed kits throughout different public spaces and hot spots in a community (flexible community model) or that targeted public spaces with high footfall (fixed-point and transport hub distribution), followed by the workplace model that used small, nimble teams and innovative management strategies, and focused on easily accessible urban workplaces such as filling stations and restaurants on the same site, as well as large urban and rural workplaces. Screening positivity was highest in the models targeting sex worker networks and partners of known HIV positives (index testing). Linkage to confirmatory testing was highest in the models that offered testing on-site, while ART initiation among HIVST users screening positive was highest in models that were situated within a facility. The lowest rates of confirmatory testing and ART initiation were seen in the standalone key populations model, possibly owing to the transitory nature of the audience which might have made it harder to direct recipients to the relevant closest clinics should they need further care.

Adding cost and comparing cost with these different outcome metrics, we were able to identify different options depending on decision makers' priorities: the sex worker and transport hubs models as well as one of the workplace models distribute kits in the most efficient and least costly way. If however the aim is to distribute kits in a way that finds the most HIV-positive people most cheaply, then secondary distribution via index cases at facility as well as sex worker network distribution are the most efficient choices.

\section{Author affiliations}

${ }^{1}$ Health Economics and Epidemiology Research Office (HE $\left.{ }^{1} \mathrm{RO}\right)$, Department of Internal Medicine, Faculty of Health Sciences, University of the Witwatersrand, Johannesburg, South Africa

${ }^{2}$ Department of Global Health and Development, London School of Hygiene and Tropical Medicine Faculty of Public Health and Policy, London, UK

${ }^{3}$ Department of HIV/AIDS and TB, Malawi-Liverpool-Wellcome Trust Clinical Research Programme, Blantyre, Malawi

${ }^{4}$ Wits Reproductive Health and HIV Research Institute, Faculty of Health Sciences, University of the Witwatersrand, Johannesburg, South Africa

${ }^{5}$ Ezintsha, Wits Reproductive Health and HIV Research Institute, University of the Witwatersrand, Johannesburg, South Africa

${ }^{6}$ Society for Family Health, Johannesburg, South Africa

${ }^{7}$ HIV Prevention Programmes, National Department of Health, Pretoria, South Africa ${ }^{8}$ Population Services International, Cape Town, South Africa

${ }^{9}$ HIV Department, World Health Organization, Geneva, Switzerland

${ }^{10} \mathrm{Joint}$ United Nations Programme on HIV/AIDS, Geneva, Switzerland

${ }^{11}$ Department of Global Health, School of Public Health, Boston University, Boston, Massachusetts, USA

Twitter Cheryl Johnson @ccasejohn

Acknowledgements We acknowledge the role played in enabling the data collection for this study by the South African Department of Health, the staff and patients of the clinics and distribution teams involved, and staff at the Wits Reproductive Health Institute (Wits RHI), Society for Family Health and Population Services International. We are grateful for the assistance of Vinolia Ntjikelane, Nonhlanhla Tshabalala and Clive Ramushu with the data collection for the timeand-motion study and the cost analysis overall.

Contributors KM, LAS, CM, MdE, FT-P and GM-R conceptualised the study and developed data collection and analytical tools. KM and GM-R wrote the first draft of the paper. KM, LAS, CM, MdE, MM, JP, VZ, CM, SK, KH, TC, CJ and FT-P contributed to the acquisition, analysis and interpretation of data. All authors revised drafts critically for important intellectual content and gave final approval of the version published.

Funding This analysis was funded through the grant 'Enhancing the evidencebase of HIV self-testing for young men' (BMGF OPP1189095) to Ezintsha, a division of Wits RHI, and HE2RO.

Competing interests None declared.

Patient consent for publication Not required.

Ethics approval Ethical approvals for the project protocol were granted by the Human Research Ethics committees of the University of the Witwatersrand (ref. M180379) and the London School of Hygiene and Tropical Medicine (ref. 15408), as well as the Institutional Review Board (IRB) of Boston University School of Public Health (IRB no. H-37713).

Provenance and peer review Not commissioned; externally peer reviewed.

Data availability statement All data relevant to the study are included in the article or uploaded as supplemental information. All data pertaining to this study are included in the paper and the supplemental materials.

Supplemental material This content has been supplied by the author(s). It has not been vetted by BMJ Publishing Group Limited (BMJ) and may not have been peer-reviewed. Any opinions or recommendations discussed are solely those of the author(s) and are not endorsed by BMJ. BMJ disclaims all liability and responsibility arising from any reliance placed on the content. Where the content includes any translated material, BMJ does not warrant the accuracy and reliability of the translations (including but not limited to local regulations, clinical guidelines, terminology, drug names and drug dosages), and is not responsible for any error and/or omissions arising from translation and adaptation or otherwise.

Open access This is an open access article distributed under the terms of the Creative Commons Attribution IGO License (CC BY NC 3.0 IGO), which permits use, distribution,and reproduction in any medium, provided the original work is properly cited. In any reproduction of this article there should not be any suggestion that WHO or this article endorse any specific organization or products. The use of the WHO logo is not permitted. This notice should be preserved along with the article's original URL.

Disclaimer: The author is a staff member of the World Health Organization. The author alone is responsible for the views expressed in this publication and they 
do not necessarily represent the views, decisions or policies of the World Health Organization.

\section{REFERENCES}

1 Johnson L. A model for evaluating the impact of HIV / AIDS in South Africa. Cent Infect Dis 2020:1-164.

2 Hansoti B, Stead D, Parrish A, et al. HIV testing in a South African emergency department: a missed opportunity. PLoS One 2018;13:e0193858.

3 Human Sciences Research Council (HSRC). HIV impact assessment summary: the fifth South African national HIV prevalence, incidence, behaviour and communication survey, 2017; 2018.

4 UNAIDS. Fast-Track - Ending the AIDS epidemic by 2030. Available: https://www.unaids.org/en/resources/documents/2014/JC2686_ WAD2014report [Accessed 10 Sep 2020].

5 World Health Organization. HIV self-Testing and partner notification supplement to consolidated guidelines on HIV testing services 2016.

6 Lyons CE, Coly K, Bowring AL, et al. Use and acceptability of HIV Self-Testing among first-time testers at risk for HIV in Senegal. AIDS Behav 2019;23:130-41.

7 Tonen-Wolyec S, Mbopi-Kéou F-X, Batina-Agasa S, et al. Acceptability of HIV self-testing in African students: a cross-sectional survey in the Democratic Republic of Congo. Pan Afr Med J 2019;33:1-6.

8 Harichund C, Moshabela M, Kunene P, et al. Acceptability of HIV self-testing among men and women in KwaZulu-Natal, South Africa. AIDS Care 2019;31:186-92.

9 Conserve DF, Muessig KE, Maboko LL, et al. Mate Yako Afya Yako: formative research to develop the Tanzania HIV self-testing education and promotion (Tanzania step) project for men. PLoS One 2018;13:e0202521.

10 Johnson LF, van Rensburg C, Govathson C, et al. Optimal HIV testing strategies for South Africa: a model-based evaluation of populationlevel impact and cost-effectiveness. Sci Rep 2019;9:1-12.
11 Mostert C. The impact and cost of HIV Self-Test distribution in workplaces in South Africa. Abstract. Conference on Retroviruses and Opportunistic Infection, 2020.

12 Maheswaran $\mathrm{H}$, Petrou S, MacPherson P, et al. Cost and quality of life analysis of HIV self-testing and facility-based HIV testing and counselling in Blantyre, Malawi. BMC Med 2016;14:34.

13 Mangenah C, Mwenge L, Sande L, et al. Economic cost analysis of door-to-door community-based distribution of HIV Self-Test kits in Malawi, Zambia and Zimbabwe. J Int AIDS Soc 2019;22(Suppl 1):e25255.

14 d'Elbée M, Makhetha MC, Jubilee M, et al. Using HIV self-testing to increase the affordability of community-based HIV testing services. AIDS 2020;34:2115-23.

15 Meehan S-A, Sloot R, Draper HR, et al. Factors associated with linkage to HIV care and TB treatment at community-based HIV testing services in Cape town, South Africa. PLoS One 2018;13:e0195208-13.

16 Vassall A, Sweeney S, Kahn JG, et al. Reference case for estimating the costs of global health services and interventions 2017.

17 Rates - South African Reserve Bank. Available: https://www.resbank. co.za/Research/Rates/Pages/Rates-Home.aspx [Accessed 05 Oct 2020].

18 "Press Release: OraSure Technologies to Drive Accelerated Adoption of OraQuick® HIV Self-Test - Gates SIF." [Online]. Available: https://sif.gatesfoundation.org/news-and-updates/ press-release-orasure-technologies-drive-accelerated-adoptionoraquick-hiv-self-test/ [Accessed 05 Oct 2020].

19 Long LC, Maskew M, Brennan AT, et al. Initiating antiretroviral therapy for HIV at a patient's first clinic visit: a cost-effectiveness analysis of the rapid initiation of treatment randomized controlled trial. AIDS 2017;31:1611-9.

20 Vassall A, Mangham-Jefferies L, Gomez GB, et al. Incorporating demand and supply constraints into economic evaluations in lowincome and middle-income countries. Health Econ 2016;25(Suppl 1):95-115. 